\title{
WRIB Poster Award winners
}

The 10th Workshop on Recent Issues in Bioanalysis (WRIB) was held in Orlando, USA, in April 2016. It drew close to 700 professionals representing large pharmas, biotechs, CROs and multiple regulatory agencies from around the world, working on both small- and large-molecule bioanalysis. This year, bioanalysis and bioanalysis zone were proud to support the WRIB Poster Awards and we feature profiles of the authors of the winning posters. Go to www.bioanalysis-zone.com to see the winning posters in full.

\section{Winning poster: Characterization of Pharmacokinetic Propertiesfor a Site-Specific Antibody Drug Conjugate (ADC) Using Multiple-Platform Bioanalysis Assays Jian Chen, Brian Melo, Matthew Myers, Martha Vallejo, Xinqun Wu, Priya Sriraman, Yongjun Xue \& Sekhar Surapanen (Drug Metabolism and Pharmacokinetics (DMPK), Non Clinical Development (NCD), Celgene Corporations, Summit, NJ, USA)}

\section{Jian Chen, Celgene Corporation, NJ, USA}

Jian Chen is currently a principal scientist working in DMPK group at Celgene. He joined Celgene in 2009 and has been responsible for supporting Celgene bioanalytical studies both in-house and at CROs. He represents bioanalytical function for several development programs in various cross-function study teams including both nonclinical and clinical studies. He is also engaged in many bioanalytical research projects conducted at DMPK Labs. His interests include microsampling techniques, bioanalytical methods for quantitation/qualification of biomarkers, protein therapeutics, antibody-drug conjugates and oligonucleotides using various LC-MS/MS platforms. Prior to joining Celgene, he worked as a research scientist at Tandem Labs, a bioanalytical CRO where he gained experiences in bioanalytical method development and validation, supported both regulated and discovery bioanalytical studies.

\section{Q What is your role at Celgene Corporation?}

I am currently working in Celgene's DMPK group as a principal scientist. I support various Celgene bioanalytical studies both inhouse and at CROs and represent bioanalytical functions on cross-function study teams. I am also actively involved in bioanalytical research from microsampling techniques to quantitation of biomarkers, protein therapeutics, antibody-drug conjugates and oligonucleotides on various LC-MS platforms.
Q What were your first thoughts on winning the 10th WRIB Poster Award?

I was quite surprised, but it is a great honor for our poster to be recognized by our peers. We also appreciate the opportunity to present our ADC research during the 10th WRIB and share our lessons learned with the bioanalytical community. Celgene has grown a lot in the past few years but our bioanalytical group is still very small compared with many other pharma companies; so, I felt very fortunate to work with such a group of highly

\section{Bioanalysis \\ Bioanalys}


innovative scientists. The award validated the tremendous efforts/contributions each coauthor made to this research and to our overall ADC program.

How did you carry out the work highlighted in this poster?

This is truly a team effort with five lab scientists working on five unique bioanalytical assays simultaneously. We started with literature research and brainstorm sessions between our LBA and MS colleagues on how to approach these assays needed to support the ADC program, followed by recurring meetings to discuss technical challenges and potential resolutions. Everyone enjoyed the learning experience, but more importantly, by overcoming these technical challenges, we were able to deliver valuable data to the study team with very tight timelines.

Q What were the key findings from this research?

A unique aspect of this research was the quantitation of total conjugated drug in serum samples by hybrid immunocapture and quantitative tryptic digestion to release playload + linker + peptide surrogate. With precursor/product scans of digested neat ADC sample, we were able to accurately predict the mass/charge states of the surrogate, and use such information to optimized
MS parameters. We characterized the linker stability for the ADC during in vivo circulation both through comparing total antibody/total conjugated antibody results and direct DAR measurement using HRMS. We also identified ADA via difference between LBA and hybrid assays and provided ADA analysis as needed.

Q What are you looking forward to working on over the next year?

We will apply the key learnings gained from this research to other ADC programs. We will also incorporate more HRMS-based assays to further characterize ADC properties in circulation such as metabolism/catabolism.

Financial \& competing interests disclosure

J Chen (and all authors who worked on the poster) are Celgene employees, the materials are from the company, and all experiments were conducted at the laboratory at Celgene. The authors have no other relevant affiliations or financial involvement with any organization or entity with a financial interest in or financial conflict with the subject matter or materials discussed in the manuscript apart from those disclosed.

No writing assistance was utilized in the production of this manuscript.

\section{Winning poster: Challenges in estimation of FSH for pharmacokinetic study by Architect i1000 (CMIA based clinical lab instrument) and commercial kits S Gupta et al.}

\section{Shaifali Gupta, Cliantha Research Limited, Ahmedabad, India}

Shaifali Gupta is the lab director at the CAP-accredited clinical reference lab and leads the LBA Lab involved with method development, validations and study sample analysis of large molecules at Cliantha Research Limited, Ahmedabad, India. She is a medical graduate with a specialization in pathology. She is also a certified NABL (National Accreditation Board of Medical Laboratories) and trained CAP assessor. Since the diagnostic lab work involves endogenous large molecules, she sees a significant overlap of work happening in the diagnostic lab arena and PK and PD work on large molecules in regulatory environment. Having worked in the clinical lab for over 15 years in the fields of clinical chemistry, immunology and hematology, there is a certain confidence on the testing of automated instruments used in the diagnostic laboratories.

Q Please can you explain about your role at Cliantha Research Limited?

At the outset, it is important to introduce Cliantha Research Limited. We are a CRO based in Ahmedabad, founded 11 years ago, involved with end-to-end services for BA/BE and CT studies. We have an analytical lab in Ahmedabad and Toronto, Canada and clinics in India and Tampa, USA.

I find myself uniquely placed to work with large molecules in regulatory environment with a vast diagnostic lab experience. We have developed a wide range of bio- marker assays on a variety of platforms including flow cytometry and clot-based assays to support the clinical trials. With the support of experienced scientists, we have also developed immunogenicity and PK methods for monoclonal antibodies and other large molecules.

\section{Q How does it feel to win the 10th WRIB Poster} Award?

We feel extremely privileged that our work has been noticed by the esteemed gathering of experts. It is highly rewarding as the attention generated by the 
award bolsters our enthusiasm to explore newer methods for large molecules.

We had initially tried to develop the method on ELISA. However, as we struggled with the sensitivity achieved and FSH molecule was already being tested by a highly sensitive method in the diagnostic lab, we planned to further develop our method on the diagnostic lab equipment using the commercial kits as per the regulatory requirements.

Recognition from the esteemed community reaffirms that this thought process needs to be explored further and used to our advantage.

Q What were the key conclusions from this research?

The most striking aspect of this study was the high sensitivity that was possible with Architecti1000 CMIA-based machine from Abbott Diagnostics.

Sensitivity is a challenge with endogenous molecules along with interferences in ELISA.

The focus of the diagnostic environment is more on safety. iVD kits may have higher sensitivity and precision required for PK/PD studies. So in order to meet the demands of the more rugged environment and regulatory guidelines, we had to work on the calibrators, controls and the software requirements.
As an afterthought, it would be great if the two communities (diagnostic and pharma industry) could interact closely. There could be a greater overlap of work as most of the biomarkers and endogenous molecules have also been explored by the diagnostic community on different platforms.

Q What plans do you have for your research over the next year?

We plan to work further on hybrid LBA/LC-MS methods, which are promising in overcoming the challenges of sensitivity and interference posed by some LBA methods. We also plan to explore molecular testing to widen our biomarkers test menu and add newer platforms for PK and immunogenicity testing of biosimilars.

\section{Financial \& competing interests disclosure}

The author has no relevant affiliations or financial involvement with any organization or entity with a financial interest in or financial conflict with the subject matter or materials discussed in the manuscript. This includes employment, consultancies, honoraria, stock ownership or options, expert testimony, grants or patents received or pending, or royalties.

No writing assistance was utilized in the production of this manuscript.

\section{Winning poster: Y Xu, Comparison of UPLC-Triple Quadrupole MS and UPLC-High Resolution MS for Human Microdosing Assays With Pico-gram per Milliliter Quantification Limit Yang Xu, David McLaren, Li Sun, Iris Xie, Irong Fu, Sheila Breidinger, Dina Goykhman, Kevin Bateman \& Eric Woolf (Merck Research Laboratories, Merck \& Co., Inc., Kenilworth, NJ, USA)}

\section{Yang Xu, Regulated Bioanalysis, PPDM, Merck \& Co., Inc., NJ, USA}

Yang Xu, is a Sr. Principal Scientist in Regulated Bioanalytics, Department of Pharmacokinetics, Pharmacodynamics \& Drug Metabolism (PPDM), at Merck Co. \& Inc. (PA, USA). She received her BS in pharmaceutical sciences from Beijing Medical University, MS in analytical chemistry from the Chinese Academy of Sciences, PhD in biochemistry from Wesleyan University and completed her postdoctoral fellowship in molecular biophysics and biochemistry at Yale University. She joined Merck in 2001 after spending 2 years at Vion Pharmaceuticals. She specializes in developing LC-MS/MS-based quantification methods for both small and large molecules to support preclinical, nonclinical GLP and clinical studies. Her research interests include evaluation of new technologies, such as microsampling (DBS, VAMS, etc.), HRMS and hybrid assays (immunoaffinity purification coupled with LC-MS/MS) for peptide/protein quantification, and their implementation into the regulated bioanalysis space.

Q Please explain your role at Merck \& Co., Inc.? I am a Sr. Principal Scientist in the Regulated Bioanalytical Method Development Group in PPDM. My main responsibility is to develop rugged analytical methods that support GLP TK and clinical PK studies for small and large molecules. In addition, I am cochair for the Microsampling Working Group in PPDM and Safety Assessment Departments to evalu- ate new microsampling devices (such as DBS card, Mitra, Noviplex, etc.). So far, DBS has been successfully implemented into our clinical studies, and the effort on Mitra implementation is on-going. Also, I am cochair for the Global Bioanalysis Peptide/Protein Quantification Team to promote sharing of our best practices and experiences on LC-MS/MS-based quantification across the Global Bioanalytics groups. 
Q How does it feel to win the 10th WRIB Poster Award?

It was an honor to receive this prize, and I truly felt that the award belongs to the group of my colleagues (coauthors) who collaborated together on this project.

Q Tell us more about the work behind your winning poster

Our main driver for comparing triple-quadrupole MS (QqQ) versus HRMS came from the presentation by Chris Evans at the 2015 WRIB meeting, demonstrating a significant sensitivity improvement using HRMS. Since our human microdosing study required supersensitive assays (LLOQ at $2 \mathrm{pg} / \mathrm{ml}$ ), we decided to explore the potential advantage of applying HRMS to optimize assay sensitivity by resolving isobaric matrix interferences. Since QqQ is currently the primary tool for quantitative bioanalysis due to its high sensitivity, a direct comparison between QqQ and HRMS (keeping sample preparation and UPLC conditions the same) could allow us to conclude the true difference between the two MS platforms, and help us understand the reasons behind our observations.

Q What conclusions can we draw from this?

Our results suggested that the sensitivity on HRMS appears to be compound dependent, and it can be comparable to that observed on QqQ MS. HRMS may be more beneficial when applied to the relatively cruder plasma extract (e.g., through protein precipitation or LLE). Its additional resolving power could improve signal-to-noise ratio, if specificity issue is encountered using QqQ.

Q What are you looking forward to working on over the next year?

The results allowed us to better understand the true benefits of HRMS, and therefore, will help us to decide when and how to use this valuable tool in our bioanalysis in the future. For example, HRMS would be a valuable alternative if we need to minimize our method development effort on sample preparation due to limited time, and a helpful tool in terms of identifying/resolving the issues with assay specificity due to matrix background or metabolite interference.

\section{Financial \& competing interests disclosure}

The author has no relevant affiliations or financial involvement with any organization or entity with a financial interest in or financial conflict with the subject matter or materials discussed in the manuscript. This includes employment, consultancies, honoraria, stock ownership or options, expert testimony, grants or patents received or pending, or royalties.

No writing assistance was utilized in the production of this manuscript. 